\title{
THE DEVIL AND THE ANGEL OF LOOPS
}

\author{
LEONG FOOK
}

\begin{abstract}
G_{a}$, the subloop generated by all the associators of a loop $G$, is singled out for study for the first time. If $G$ is Moufang, $G_{a}$ is found to be normal in $G$. The relation of $G_{a}$ with the nucleus of $G$ is also investigated.
\end{abstract}

A binary system $(G, \cdot)$ is a loop if (i) $(G, \cdot)$ is closed, (ii) $(G, \cdot)$ has an identity 1, (iii) $x, y \in G \Rightarrow$ there exist unique $u, v \in G$ such that $x u=y, v x=y$.

A group is a loop; but a loop may not be a group. The difference lies on the Associative Law.

For $x, y, z \in G$, we can write $x y \cdot z=(x \cdot y z)(x, y, z) .(x, y, z)$ is called the associator of $x, y, z$. The subloop $G_{a}$, generated by all the associators of $G$, is called the associator subloop of $G$. If $G_{a}=1$, then $G$ is obviously a group and everything will be fine. On the other hand, if $G_{a}$ is nontrivial, the loop may be so difficult that even the best genius will fight shy of it. It is therefore not inappropriate to call $G_{a}$ the devil of $G$.

In contrast with this, we have the nucleus $N$ of $G . N \subset G$ and for any $n \in N,(n, x, y)=(x, n, y)=(x, y, n)=1$ for all $x, y \in G$. Clearly $N$ is a group. It helps us as a stepping stone to understand the loop $G$. It is therefore not inappropriate to call $N$ the angel of $G$.

For an arbitrary loop $G$, nothing much can be said about the devil and the angel. If $G$ is Moufang, i.e., $x y \cdot z x=(x \cdot y z) x$ for all $x, y, z \in G$, then they become beautiful:

THeOrem 1. If $G$ is a Moufang loop, then $N \triangleleft G$.

Proof. By [1, p. 114, Theorem 2.1] and by disassociativity of $G$.

We wish to investigate if the devil also possesses this property:

Definition. Let $(G, \cdot)$ be a loop. $A(x, y, z)$ and $B(x, y, z)$ are defined as:

(a) $(x y) z=[A(x, y, z) \cdot x](y z)$;

(b) $x(y z)=[(B(x, y, z) \cdot x) \cdot y] \cdot z$ for all $x, y, z \in G$.

TheOREM 2. Let $(G, \cdot)$ be a loop; $H=\langle A(x, y, z), B(x, y, z) \mid x, y, z \in G\rangle$. Then (i) $(H x) y=H(x y), H[(x y) z]=H[x(y z)]$ for all $x, y, z \in G$, (ii) $H$ is the smallest normal subloop of $G$ such that $G / H$ is a group.

Proof. (i) By (a), with $x=h \in H,(h y) z \in H(y z)$ for all $h \in H$. Therefore $(H y) z \subset H(y z)$ for all $y, z \in G$. By (b), taking $x=h \in H, h(y z)$ $\in(H y) \cdot z$ for all $h \in H$. Therefore $H(y z) \subset(H y) \cdot z$ for all $y, z \in G$. So $(H x) y=H(x y)$ for all $x, y \in G$.

Received by the editors December 5, 1974.

AMS (MOS) subject classifications (1970). Primary 20 N05.

Key words and phrases. Loop, associator, Moufang. 
By (a), $x y \cdot z \in(H x)(y z)$. But $(H x)(y z)=H(x \cdot y z)$. Hence $(x y) z=h_{1}$ - $x(y z)$ for some $h_{1} \in H$. Then

$$
H((x y) z)=H\left[h_{1} \cdot(x \cdot y z)\right]=\left(H h_{1}\right) \cdot(x \cdot y z)=H \cdot(x \cdot y z) .
$$

(ii) For each $x \in G$, define the map $\alpha_{x}$ by $(H y) \alpha_{x}=(H y) \cdot x$ for all $y \in G$. It is easy to verify that $\alpha_{x}$ is a permutation of the set of right cosets $H y, y \in G$.

For each $x \in G$, define $\alpha$ by $x \alpha=\alpha_{x}$. Then for all $x, y, z \in G$,

$$
\begin{aligned}
(H x) \alpha_{y} \alpha_{z} & =[(H x) y] \cdot \alpha_{z}=(H(x y)) \cdot z=H(x y \cdot z) \\
& =H(x(y z))=(H x) \alpha_{y z} .
\end{aligned}
$$

Therefore $\alpha_{y} \alpha_{z}=\alpha_{y z}$ or $(y z) \alpha=y \alpha z \alpha$ for all $y, z \in G$. So $\alpha$ is a homomorphism of $(G, \cdot)$ into a permutation group. $x \in \operatorname{ker}(\alpha) \Leftrightarrow(H y) x=H y$ for all $y$ $\in G$. Taking $y \in H$, we have $H x=H$ or $x \in H$. So $\operatorname{ker}(\alpha) \subset H$. As $G / \operatorname{ker}(\alpha)$ is a group, it can be seen easily that $\operatorname{ker}(\alpha)$ contains $A(x, y, z)$ and $B(x, y, z)$ for all $x, y, z \in G$. So $\operatorname{ker}(\alpha) \supset H$. Hence, $H=\operatorname{ker}(\alpha)$.

Let $S$ be another normal subloop of $G$ such that $G / S$ is a group. Then

$$
(x S \cdot y S) z S=[A(x, y, z) S \cdot x S] \cdot[y S \cdot z S] .
$$

By cancellation, we have $A(x, y, z) \in S$. Similarly $B(x, y, z) \in S$. Thus $H \subset S$.

COROllary. Let $G$ be a Moufang loop. Then $G_{a}$ is the smallest normal subloop of $G$ such that $G / G_{a}$ is a group.

Proof. Let $x, y, z \in G$. By [1, p. 124, Lemma 5.4 (5.13) and (5.16)], $z R(x, y)=z L\left(x^{-1}, y^{-1}\right)=z\left(z, y^{-1}, x^{-1}\right)^{-1}$. Similarly, we have $z^{-1} R(x, y)$ $=z^{-1}\left(z^{-1}, y^{-1}, x^{-1}\right)^{-1}$. As $R(x, y)$ is a pseudoautomorphism of $G, z^{-1} R(x, y)$ $=(z R(x, y))^{-1}$. Thus $z R(x, y)=\left(z^{-1}, y^{-1}, x^{-1}\right) \cdot z$.

By [1, p. 124, Lemma 5.4, (5.13) and (5.16)], $z R(x, y)^{-1}=z L\left(y^{-1}, x^{-1}\right)$ $=z\left(z, x^{-1}, y^{-1}\right)^{-1}$. Thus $z^{-1} R(x, y)^{-1}=z^{-1}\left(z^{-1}, x^{-1}, y^{-1}\right)^{-1}$. But $z^{-1} R(x, y)^{-1}=\left[z R(x, y)^{-1}\right]^{-1}$. Therefore $z R(x, y)^{-1}=\left(z^{-1}, x^{-1}, y^{-1}\right) z$.

By definition of $A(x, y, z)$ and $B(x, y, z), z R(x, y)=A(z, x, y) \cdot z \quad$ and $z R(x, y)^{-1}=B(z, x, y) \cdot z$. Therefore $A(z, x, y)=\left(z^{-1}, y^{-1}, x^{-1}\right), B(z, x, y)$ $=\left(z^{-1}, x^{-1}, y^{-1}\right)$. So

$$
\begin{aligned}
H & =\langle A(z, x, y), B(z, x, y) \mid x, y, z \in G\rangle \\
& =\left\langle\left(z^{-1}, y^{-1}, x^{-1}\right),\left(z^{-1}, x^{-1}, y^{-1}\right) \mid x, y, z \in G\right\rangle=G_{a} .
\end{aligned}
$$

THEOREM 3. Let $G$ be a loop and $H$ a normal subloop of $G$ such that $H \leqq N$. Then:

(a) $G / C_{G}(H) \leqq$ Aut $H$ where $C_{G}(H)=\{g \mid g \in G, h g=g h$, for all $h$ $\in H\}$.

(b) $C_{G}(H) \cap H=Z(H)$, the center of $H$.

Proof. Let $x \in G$. Define $T_{x}$ by means of $h x=x \cdot h T_{x}$ for all $h \in H$. By normality $H x=x H$. Thus, $T_{x}$ is a permutation of $H$. If $h, h^{\prime} \in H$, then, 


$$
\begin{aligned}
x\left(\left(h h^{\prime}\right) T_{x}\right) & =\left(h h^{\prime}\right) x & & \text { by definition, } \\
& =h\left(h^{\prime} x\right) & & \text { as } h^{\prime} \in H \subset N, \\
& =h\left(x\left(h^{\prime} T_{x}\right)\right) & & \text { by definition, } \\
& =h x \cdot\left(h^{\prime} T_{x}\right) & & \text { since } h^{\prime} T_{x} \in H \subset N, \\
& =\left(x \cdot h T_{x}\right)\left(h^{\prime} T_{x}\right) & & \text { by definition, } \\
& =x\left(h T_{x} \cdot h^{\prime} T_{x}\right) & & \text { as } h^{\prime} T_{x} \in H \subset N .
\end{aligned}
$$

Therefore, $\left(h h^{\prime}\right) T_{x}=h T_{x} \cdot h^{\prime} T_{x}$ for all $h, h^{\prime} \in H$, for all $x \in G . T_{x}$ is an automorphism of $H$ for each $x \in G$.

Consider the map $x \rightarrow T_{x}, x \in G$. Let $y \in G$. Then for all $h \in H$,

$$
\begin{aligned}
(x y)(h T(x y)) & =h(x y) & & \text { by definition, } \\
& =h x \cdot y & & \text { as } H \leqq N, \\
& =x\left(h T_{x}\right) \cdot y & & \text { by definition, } \\
& =x \cdot h T_{x} y & & \text { as } h T_{x} \in H \subset N, \\
& =x\left(y\left(h T_{x} T_{y}\right)\right) & & \text { by definition, } \\
& =(x y)\left(h T_{x} T_{y}\right) & & \text { as } h T_{x} T_{y} \in N .
\end{aligned}
$$

Thus $h T_{x y}=h T_{x} T_{y}$ and $T_{x y}=T_{x} T_{y}$ for all $x, y \in G$. So, $\alpha$ is a homomorphism of $G$ into $\operatorname{Aut}(H)$.

Let $x \in G . T_{x}=I \Leftrightarrow h x=x h$ for all $h \in H$. Therefore, the kernel is $C_{G}(H)$ and (a) is proven.

Since $H \subset N, H$ is a group. So (b) is clear.

Corollary. Let $G$ be a Moufang loop. Then $G_{a} \subset C_{G}(N)$.

Proof. Let $H=N$ in the Theorem. As $G / C_{G}(N)$ is a group, $G_{a} \subset C_{G}(N)$.

The relations between the angel and the devil in the Moufang case are as shown in the diagram:

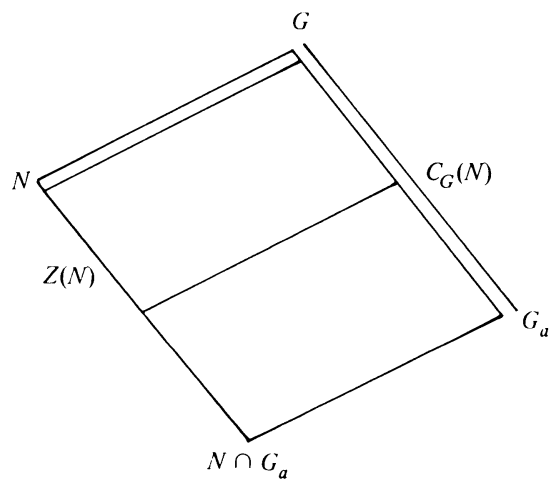

REFERENCE

1. R. H. Bruck, A survey of binary systems, Springer-Verlag, New York, 1971. 